\title{
Erratum to: A case of Klinefelter syndrome with aplastic anemia
}

\author{
Chao Xu $\cdot$ Cai-Yu Zhang $\cdot$ Nan Chen • \\ Xun Sun • Ye Xiao • Ling Gao • Jia-Jun Zhao
}

Published online: 4 March 2011

(C) The Japanese Society of Hematology 2011

Erratum to: Int J Hematol (2011) 93:213-215

DOI 10.1007/s12185-011-0767-1

This article originally was published without including the following Acknowledgment:

This work was supported by grants from the National Natural Science Foundation of China (81000039).

The online version of the original article can be found under doi:10.1007/s12185-011-0767-1.

C. Xu $(\bowtie) \cdot$ J.-J. Zhao

Department of Endocrinology and Metabolism, Provincial Hospital Affiliated to Shandong University, Institute of Endocrinology, Shandong Academy of Clinical Medicine, 324 Jing 5 Rd., Jinan 250021, Shandong, China e-mail: doctorxuchao@163.com

C. Xu · C.-Y. Zhang $\cdot$ X. Sun $\cdot$ Y. Xiao

Weihai Hospital, An Affiliate of Medical College of Qingdao University, Weihai 264200, Shandong, China

N. Chen

Department of Dermatology, Shandong Provincial Hospital

Affiliated to Shandong University, Jinan, China

L. Gao

Department of Scientific Center, Provincial Hospital

Affiliated to Shandong University, 324 Jing 5 Rd.,

Jinan 250021, Shandong, China 